Draft Version July 16, 2021

Preprint typeset using $\mathrm{LAT}_{\mathrm{E} X} \mathrm{X}$ style emulateapj v. 5/2/11

\title{
DYNAMICAL MODEL OF AN EXPANDING SHELL
}

\author{
ASAF PE'ER ${ }^{1}$ \\ Draft version July 16, 2021
}

\begin{abstract}
Expanding blast waves are ubiquitous in many astronomical sources, such as supernovae remnants (SNRs), X-ray emitting binaries (XRBs) and gamma-ray bursts (GRBs). I consider here the dynamics of such an expanding blast wave, both in the adiabatic and the radiative regimes. As the blast wave collects material from the surrounding, it decelerates. A full description of the temporal evolution of the blast wave requires consideration of both the energy density and the pressure of the shocked material. The obtained equation is different than earlier works in which only the energy was considered. The solution converges to the familiar results in both the ultra-relativistic and the sub-relativistic (Newtonian) regimes.
\end{abstract}

Subject headings: hydrodynamics — ISM: jets and outflows — relativity — shock waves

\section{INTRODUCTION}

Expanding blast waves are one of the most common phenomena in many astronomical transients. Interaction of the expanding shells of supernova remnants (SNRs) with their environment was studied more than three decades ago (Chevalier 1976, 1982). Similarly, in X-ray emitting binaries (XRBs), mildly relativistic (Lorentz factor $\Gamma \sim$ few) expanding radio blobs are observed for nearly two decades now (Mirabel \& Rodríguez 1994; Hjellming \& Rupen 1995; Fender et al. 1999). In gamma-ray bursts (GRBs) relativistic blast waves are an inherent part of the GRB "fireball" model (Goodman 1986; Paczynski 1986; Shemi \& Piran 1990; Rees \& Meszaros 1992; Naravan et al. 1992), and are the source of the afterglow emission frequently seen.

These expanding blast waves originate from a stellar explosion (such as in GRBs or supernovae), or a rapid ejection of material (as in XRBs). As they propagate through the ambient medium, they collect material and decelerate. The expansion may be adiabatic, or highly radiative. The dynamics of the blast wave expansion in both these scenarios were extensively studied in the past (Blandford \& McKee 1976; Katz \& Piran 1997; Chiang \& Dermer 1999; Piran 1999; Huang et al. 1999; van Paradijs et al. 2000) 2 These formulations are used

\footnotetext{
${ }^{1}$ Harvard-Smithsonian Center for Astrophysics, MS-51, 60 Garden Street, Cambridge, MA 02138, USA

2 While Blandford \& McKee (1976) provided a full solution in the ultra-relativistic, adiabatic limit, the original equation that describes the blast wave evolution in the radiative scenario was written and solved by Katz \& Piran (1997). It was generalized to include both the radiative and adiabatic scenarios by Chiang \& Dermer (1999); Piran (1999). However, it was later shown by Huang et al. (1999) that this equation does not converge to the correct asymptotic solution for adiabatic expansion in the Newtonian limit. Huang et al. (1999) thus modified this equation, solved the revised equation and showed that its solution converges to the correct asymptotic solutions in both the ultrarelativistic and the Newtonian limits, for both adiabatic and radiative scenarios. Bianco \& Ruffini (2004, 2005a]b), apparently being unaware of the work of Huang et al. (1999), have re-solved the dynamical equation written by Chiang \& Dermer (1999); Piran (1999), both numerically and analytically. Apart from the work by Blandford \& McKee (1976), all the other works relied on similar basic assumptions, in which the contribution of the shocked-ISM pressure was neglected, and are therefore conceptually incorrect.
}

until today as a basis for calculating the expected radiation during the deceleration phase of the blast wave evolution (e.g., Naravan et al. 2011; Granot \& Piran 2012; van Eerten \& MacFadven 2012; Shen \& Matzner 2012).

The dynamical calculations in the above mentioned works are based on a "toy model", in which a basic assumption is that the interaction between the blast wave and the interstellar material (ISM) can be described by a series of inelastic collisions (Katz \& Piran 1997; Huang et al. 1999). While this prescription asymptotes to the known analytical solutions in the ultra-relativistic limit $\Gamma \gg 1$ (Meszaros \& Rees 1997) and in the nonrelativistic limit (Sedov 1959), it neglects the contribution of the swept-up material to the internal pressure. This, in turn, affects the evolution of the blast wave dynamics. In this letter, I revise the basic assumptions of the blast wave - ISM interaction scenario, and re-derive the equations that govern the blast wave evolution. As will be shown below, the obtained equations are different than the ones previously used. Nonetheless, they asymptote to the known results at the ultra-relativistic as well as the sub-relativistic limits.

\section{DYNAMICAL MODEL}

Consider an explosion that ejects mass $M$ and creates a shell that propagates into the cold ISM. In front of the expanding shell is a blast wave. The system is thus composed of 3 regions: (1) the unshocked ISM, (2) the shocked ISM, and (3) the ejected shell material. For simplicity, I assume that a reverse shock wave (if created) had long passed, hence the ejected shell material is cold. Furthermore, I assume that the thermodynamical quantities of the gas: $n_{i}, p_{i}$ and $e_{i}$ (particle number density, pressure and internal energy density) are steady in each region. The pressure in each region is given by $p^{\prime}=(\hat{\gamma}-1)\left(e^{\prime}-\rho^{\prime}\right)$, where $\rho^{\prime}=n^{\prime} m_{p} c^{2}$ is the rest mass density and $\hat{\gamma}$ is the adiabatic index. Here and below, primed quantities are in the comoving frame, and unprimed quantities are in the observer's frame in which the unshocked ISM is at rest.

In the following, I will compare the results derived here to the results derived by Huang et al. (1999), that were shown to converge to the correct asymptotic limits. 
The evolution of the blast wave as it propagates through the ISM is calculated by considering energy conservation in the observer's frame. Let us assume that at time $t$ the plasma propagates with Lorentz factor $\Gamma 3 \mathrm{Ne}$ glecting radiative losses and assuming that the gas can be described as a prefect fluid, the energy density in region (2) as is measured by a distant observer is given by

$$
\begin{aligned}
e_{2}=T^{00} & =\left(e_{2}^{\prime}+p_{2}^{\prime}\right) u^{0} u^{0}+p_{2}^{\prime} g^{00} \\
& =\left[\hat{\gamma} \Gamma^{2}-(\hat{\gamma}-1)\right] e_{2}^{\prime}+(\hat{\gamma}-1)\left(1-\Gamma^{2}\right) n_{2}^{\prime} m_{p} c^{2}
\end{aligned}
$$

Here, $T^{00}$ is the 00 component of the stress-energy tensor. Due to Lorentz contraction, the comoving volume of region (2) is $V^{\prime}=\Gamma V$. The total energy of the gas contained in this region (in the adiabatic case), as is viewed by a distant observer is thus

$$
\begin{aligned}
E_{2}(\text { ad. }) & =\left[\hat{\gamma} \Gamma^{2}-(\hat{\gamma}-1)\right] \frac{e_{2}^{\prime} V^{\prime}}{\Gamma}+(\hat{\gamma}-1)\left(1-\Gamma^{2}\right) \frac{n_{2}^{\prime} m_{p} c^{2} V^{\prime}}{\Gamma} \\
& =\left[\hat{\gamma} \Gamma^{2}-(\hat{\gamma}-1)\left(1+\Gamma \beta^{2}\right)\right] N_{2} m_{p} c^{2}
\end{aligned}
$$

where $N_{2}=n_{2} V$ is the number of particles in region (2), $\beta \equiv\left(1-\Gamma^{-2}\right)^{1 / 2}$ is the normalized bulk velocity of the plasma in this region, and use was made in the relation $e_{2}^{\prime} / n_{2}^{\prime}=\Gamma m_{p} c^{2}$, which is exact for any value of $\Gamma$ as long as the unshocked ISM in region (1) is cold (Blandford \& McKee 1976).

The calculation in equation 2 assumes no radiative losses. In order to allow the possibility that part of the thermal energy gained by the ISM as it crosses the shock wave is radiated, equation 2 is modified as follows. The energy calculated in equation 2 is the sum of three separate components: (1) the rest mass energy of the shocked ISM; (2) its kinetic energy; and (3) its thermal energy. The first two components sum up to $\Gamma N_{2} m_{p} c^{2}$. Only the energy in the third component can in principle be radiated. If a fraction $\epsilon$ of the thermal energy is radiated, then the energy of the gas in region (2) is given by

$$
E_{2}=\left\{\Gamma+(1-\epsilon)\left[\hat{\gamma} \Gamma^{2}-\Gamma-(\hat{\gamma}-1)\left(1+\Gamma \beta^{2}\right)\right]\right\} m c^{2},
$$

where $m \equiv N_{2} m_{p}$ is the mass of the shocked ISM. The material in region (3) is assumed to be cold, and so its (kinetic + rest mass) energy is $E_{3}=\Gamma M c^{2}$.

A differential equation for the evolution of the plasma velocity is derived in the following way. Between times $t$ and $t+\delta t$, the plasma propagates a distance $\beta c \delta t$, and an ISM of mass $d m$ crosses the forward shock and gains kinetic and thermal energy. A fraction $\epsilon$ of the gained thermal energy is assumed to be radiated, hence the radiated energy is $\delta E_{\text {rad }}=\epsilon\left[\hat{\gamma} \Gamma^{2}-\Gamma-(\hat{\gamma}-1)\left(1+\Gamma \beta^{2}\right)\right] d m c^{2}$. As it collects material, the plasma decelerates; at time $t+\delta t$ its Lorentz factor is $\Gamma-d \Gamma$ (corresponding velocity $\beta-d \beta)$. Conservation of energy at times $t$ and $t+\delta t$ is

\footnotetext{
${ }^{3}$ Note that the calculation here is general, and is not limited to ultra-relativistic speeds, where $\Gamma \gg 1$.
}

written as

$$
\begin{aligned}
& \left\{\Gamma+(1-\epsilon)\left[\hat{\gamma} \Gamma^{2}-\Gamma-(\hat{\gamma}-1)\left(1+\Gamma \beta^{2}\right)\right]\right\} m+\Gamma M \\
& =-d m+\left\{(\Gamma-d \Gamma)+(1-\epsilon)\left[\hat{\gamma}(\Gamma-d \Gamma)^{2}-(\Gamma-d \Gamma)\right.\right. \\
& \left.\left.-(\hat{\gamma}-1)\left(1+(\Gamma-d \Gamma)(\beta-d \beta)^{2}\right)\right]\right\}(m+d m) \\
& +(\Gamma-d \Gamma) M+\epsilon\left[\hat{\gamma}(\Gamma-d \Gamma)^{2}-(\Gamma-d \Gamma)-\right. \\
& \left.(\hat{\gamma}-1)\left(1+(\Gamma-d \Gamma)(\beta-d \beta)^{2}\right)\right] d m \text {. }
\end{aligned}
$$

Re-arranging the terms in equation 4, it can be written as a dynamical equation for the evolution of the bulk motion Lorentz factor,

$$
\frac{d \Gamma}{d m}=-\frac{\hat{\gamma}\left(\Gamma^{2}-1\right)-(\hat{\gamma}-1) \Gamma \beta^{2}}{M+\epsilon m+(1-\epsilon) m\left[2 \hat{\gamma} \Gamma-(\hat{\gamma}-1)\left(1+\Gamma^{-2}\right)\right]} .
$$

Equation 5 thus describes the evolution of the plasma Lorentz factor due to its interaction with the ISM. This equation holds both in the ultra-relativistic $(\Gamma \gg 1)$ as well as the sub-relativistic $(\beta \ll 1)$ limits. It can be compared to equation (7) in Huang et al. (1999), which, as described above, was derived based on the assumption of continuous inelastic collisions between the blast wave and the ISM, and hence does not contain the contribution of the shock-heated ISM to the pressure in region (2) 4

\subsection{Asymptotic limits}

It is possible to obtain analytical solutions to the dynamical equation 5 in the limits of ultra-relativistic and sub-relativistic limits. These are useful for comparison with former results.

In the adiabatic scenario, $\epsilon=0$, and equation 5 reduces to

$$
\frac{d \Gamma}{d m}=-\frac{\hat{\gamma}\left(\Gamma^{2}-1\right)-(\hat{\gamma}-1) \Gamma \beta^{2}}{M+m\left[2 \hat{\gamma} \Gamma-(\hat{\gamma}-1)\left(1+\Gamma^{-2}\right)\right]} .
$$

In the ultra-relativistic limit, $\Gamma \gg 1$, equation 6 can be re-written as

$$
\frac{d \Gamma}{d m} \simeq-\frac{\left(\Gamma^{2}-1\right)}{(M / \hat{\gamma})+2 \Gamma m}
$$

Denoting by $\Gamma_{0}$ the initial Lorentz factor of the flow, the evolution of the plasma Lorentz factor can be separated into two regimes. (1) Initially, $M / \hat{\gamma} \gg \Gamma_{0} m$. In this regime, $\Gamma \simeq \Gamma_{0}$. (2) For $\Gamma m \gg M / \hat{\gamma}$ one obtains the familiar solution, $\Gamma \propto m^{-1 / 2}$, which, for constant density ISM $\left(n \propto r^{0}, m \propto r^{3}\right)$ results in the well known solution $\Gamma \propto r^{-3 / 2}$. 5 Interestingly, equation 7 is similar to equation 8 of Huang et al. (1999), with $M$ replaced by $M / \hat{\gamma} \simeq(3 / 4) M$. This discrepancy has only a minor effect on the blast wave evolution.

On the other extreme, the sub-relativistic limit $\beta \ll 1$, equation 6 reduces to

$$
\frac{d(\Gamma \beta)}{d m} \simeq-\frac{\beta}{M+m\left[2-\beta^{2}\right]},
$$

which admits the solution $\beta \propto m^{-1 / 2}$, for $m \gg M$. Thus, the general solution describing the blast wave evolution in the adiabatic scenario (equation 6) for $\Gamma m \gg$ $M / \hat{\gamma}$ can be written as $\Gamma \beta \propto m^{-1 / 2}$.

${ }^{4}$ Note that equation (7) of Huang et al. (1999) is obtained by setting $\hat{\gamma}=1$ in equation 5

${ }^{5}$ Equation 17 does not admit a third regime, $\Gamma_{0} m \gg M \gg \Gamma m$. 
In the radiative scenario, $\epsilon=1$. In the ultra-relativistic limit $\Gamma \gg 1$, the dynamical equation (eq. 5) becomes

$$
\frac{d \Gamma}{d m} \simeq-\frac{\hat{\gamma} \Gamma^{2}}{M+m} .
$$

Initially, $M \gg m$, resulting in a steady Lorentz factor, $\Gamma \simeq \Gamma_{0}$. At a later stage, $\Gamma_{0} m \gg M$ and one obtains the decay law $\Gamma(m) \simeq M / \hat{\gamma} m$ (as long as $m \ll M)$. For constant density ISM, $n \propto r^{0}$, this leads to the familiar decay law, $\Gamma \propto r^{-3}$. This result is different than the result of Huang et al. (1999) by a factor $(\hat{\gamma})^{-1} \simeq 3 / 4$.

In the sub-relativistic limit, $\beta \ll 1$, equation 5 becomes

$$
\frac{d(\Gamma \beta)}{d m} \simeq-\frac{\beta}{M+m},
$$

with the solution $\beta \propto m^{-1}$ (for $m \gg M$ ). This solution is similar to the classical "snowplow" evolution of an expanding supernova remnants in the radiative regime (Spitzer 1968).

\section{NUMERICAL SOLUTION}

A full solution of the dynamic equation 5 can easily be obtained numerically. In solving this equation, one first needs to determine the value of the adiabatic index $\hat{\gamma}$, which depends on the gas temperature. In calculating $\hat{\gamma}$, I assume that the gas in region (2) maintains a Maxwellian distribution with normalized temperature $\theta \equiv k_{B} T / m_{p} c^{2}$. The average energy per particle in this region is thus given by $<e_{2}^{\prime} / n_{2}^{\prime} m_{p} c^{2}>=$ $K_{1}\left(\theta^{-1}\right) / K_{2}\left(\theta^{-1}\right)+3 \theta$. Here, $K_{1}, K_{2}$ are modified Bessel $K$-functions of the second kind (Lightman et al. 1975, $5.34)$.

The ratio $<e_{2}^{\prime} / n_{2}^{\prime} m_{p} c^{2}>=\Gamma$ is determined by the shock jump conditions, and is thus known at any given instance. For a given $\Gamma$, a good fit to the normalized temperature is

$$
\theta \simeq\left(\frac{\Gamma \beta}{3}\right)\left(\frac{\Gamma \beta+1.07(\Gamma \beta)^{2}}{1+\Gamma \beta+1.07(\Gamma \beta)^{2}}\right) .
$$

This fit asymptotes to the exact solution in the limits $\Gamma \gg 1$ and $\beta \ll 1$. The maximum error found is less than $3 \times 10^{-3}$, for $\Gamma \beta \simeq 1$. Once the gas temperature is calculated, I use the polynomial fit given by Service (1986) , to calculate $\hat{\gamma} \simeq\left(5-1.21937 z+0.18203 z^{2}-\right.$ $\left.0.96583 z^{3}+2.32513 z^{4}-2.39332 z^{5}+1.07136 z^{6}\right) / 3$, where $z \equiv \theta /(0.24+\theta)$. This fit is accurate to $10^{-3}$.

Equation [5] is solved using 4th order Runge-Kutta method. I consider two sets of parameters, one representing GRB and one XRB. In both scenarios, the blast wave is assumed to propagate into a constant density ISM, and hence the collected mass is

$$
d m=4 \pi r^{2} n m_{p} d R,
$$

where $n$ is the number density of the ISM and $m_{p}$ is the proton rest mass. Photons emitted as the plasma propagates a distance $d R$ are observed at time $d t$, given by

$$
d R=\Gamma \beta c(\Gamma+\Gamma \beta) d t .
$$

While equation 13 is derived under the assumption that the observed photons are emitted from a plasma which propagates towards the observer, a more comprehensive

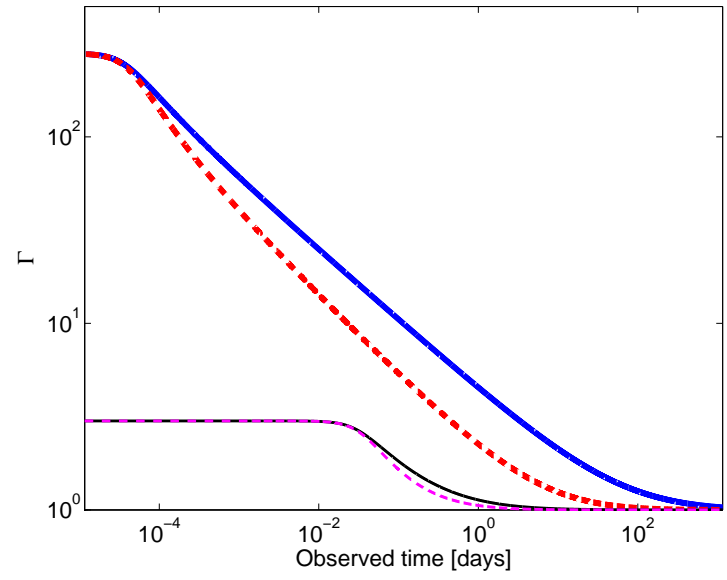

FIG. 1.- Temporal evolution of the bulk Lorentz factor, $\Gamma$. Thick lines are for "GRB" scenario $\left(\Gamma_{0}=278, M=4 \times 10^{28} \mathrm{gr}\right)$, while thin lines are for "XRB" scenario $\left(\Gamma_{0}=3, M=3 \times 10^{23} \mathrm{gr}\right)$. In all cases, ISM density $n=1 \mathrm{~cm}^{-3}$ assumed. Solid: adiabatic, $\epsilon=0$ (blue, black: GRB, XRB respectively); Dashed: radiative, $\epsilon=1$ (red, magenta: GRB, XRB respectively).

calculation which considers the integrated emission from different angles to the line of sight results in a similar solution, up to a numerical factor of a few (Waxman 1997; Pe'er \& Wijers 2006).

The evolution of the Lorentz factor $\Gamma$, the momentum $\Gamma \beta$ and the radius $R$ are presented in figures 1- -3 , In solving the dynamical equation, an ISM density of $n=$ $1 \mathrm{~cm}^{-3}$ is taken. For the initial explosion conditions, two sets of parameters are used. The first set is representative for GRBs: I take $E=10^{52} \mathrm{erg}$ and $M=2 \times 10^{-5} M_{\odot}$, resulting in $\Gamma_{0}=278$ (Huang et al. 1999). The second set is representative for XRBs, and is chosen as follows. As an initial Lorentz factor I take a fiducial value $\Gamma_{0}=3$ (Miller-Jones et al. 2005). Observed XRB radio blobs are typically emitted when the luminosity is close to the Eddington luminosity, and the flux rise time is a few days, hence the total energy released is of the order of $\sim 10^{45} \mathrm{erg}$ (e.g., Fender et al. 2004). With $\Gamma_{0}=3$ this leads to an ejected mass of $M=3 \times 10^{23} \mathrm{gr}$.

The results in Figures 1- 3 are given for both the adiabatic scenario and the radiative scenario. As expected, the results asymptote to the known solutions, which can be divided into 3 regimes. (I) Initially, $\Gamma \simeq \Gamma_{0}$, and $R \propto t$; (II) $\Gamma m \gg M$, and $\Gamma \gg 1$. In the adiabatic scenario, this leads to $R(t) \propto t^{1 / 4}$ and $\Gamma(t) \propto t^{-3 / 8}$, while in the radiative scenario, $R(t) \propto t^{1 / 7}$ and $\Gamma(t) \propto t^{-3 / 7}$. (III) For $\beta \ll 1$, in the adiabatic scenario $R(t) \propto t^{2 / 5}$ and $\beta(t) \propto t^{-3 / 5}$, while in the radiative scenario, $R(t) \propto t^{1 / 4}$ and $\beta(t) \propto t^{-3 / 4}$.

\section{SUMMARY AND DISCUSSION}

In this letter, I have revisited the dynamics of a blast wave propagating into an ISM, as is expected in many astronomical objects, such as GRBs, XRBs and Supernovae. I derived an equation (5) that determines the evolution of the blast wave Lorentz factor as the plasma collects material from the ISM and decelerates. Analytical solutions in both the adiabatic and the radiative regimes are found in the asymptotic limits $\Gamma \gg 1$ and $\beta \ll 1$ (2.1). Numerical integration is easily carried, and the resulting dynamics valid in the full regime is 


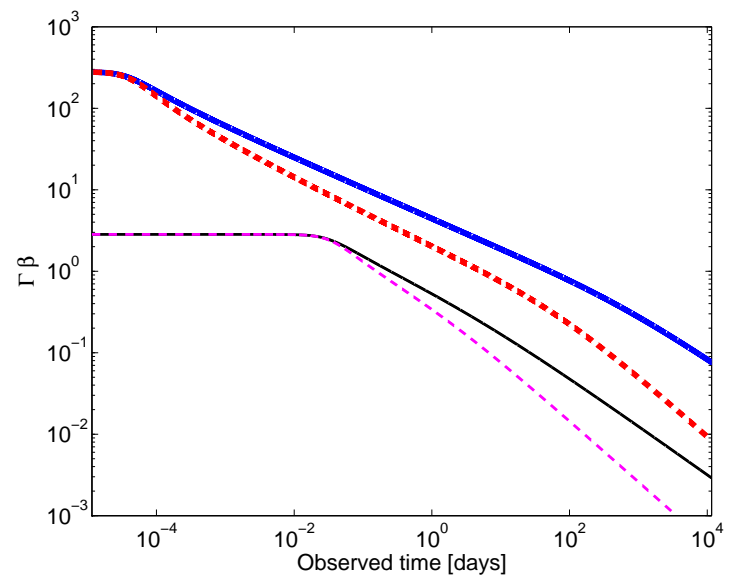

FIG. 2.- Temporal evolution of the momentum, $\Gamma \beta$. All parameters values are similar to Figure 1

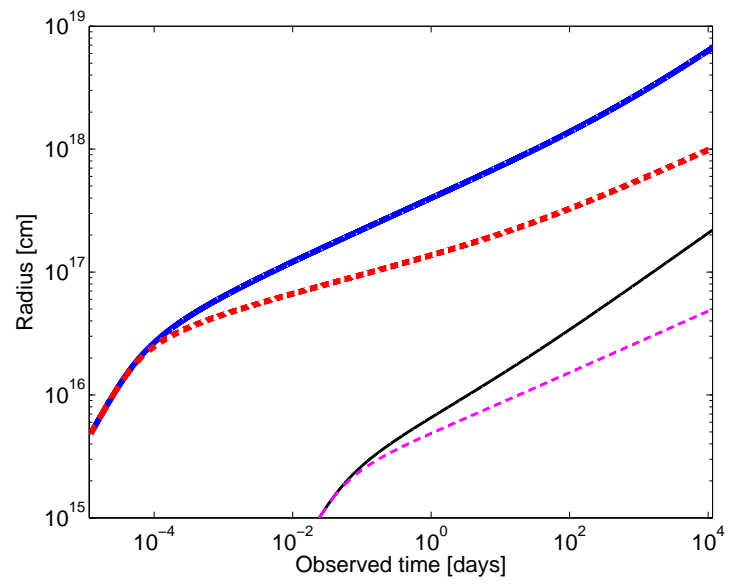

FIG. 3.- Temporal evolution of the shock radius, $R$. All parameters values are similar to Figure 1

presented 93 .

The dynamical equation is different than the dynamical equations derived earlier by several authors (Katz \& Piran 1997; Huang et al. 1999; Chiang \& Dermer 1999; Piran 1999). This is due to a conceptual difference: earlier works considered a "toy model", in which the basic assumption is that the interaction between the blast wave and the interstellar material (ISM) can be described by a series of inelastic collisions. As opposed to that, here I consider the full energy-momentum tensor, which takes into account the contribution of the collected material to both the energy and the pressure in the shocked region. Such an inclusion was neglected in earlier works.

While the results of earlier works retrieve the correct asymptotic behavior in the limits $\Gamma \gg 1$ and $\beta \ll 1$ (Huang et al. 1999), the evolution of the Lorentz factor derived in these works is different than the evolution derived here by a numerical factor of tens of percents. In the radiative scenario, the difference in the derived value of the Lorentz factor at a given radius is up to $\gtrsim 30 \%$. This result is not surprising, given that the main difference between the dynamics derived here and the dynamics derived in former works lies in the inclusion of $\hat{\gamma} \simeq 4 / 3$ (for $\Gamma \gg 1$ ). Interestingly, also in the adiabatic scenario the numerical difference is larger than $12 \%$.

In recent years, a renewed interest in calculating the expected flux from the interaction of an expanding blast wave with its environment had emerged. Works had been carried in the context of GRB afterglow emission (Granot \& Ramirez-Ruiz 2010; Granot \& Piran 2012; van Eerten \& MacFadyen 2012; Xu et al. 2012), the evolution of the observed radio blobs seen in XRBs (Shen \& Matzner 2012; Narayan \& McClintock 2012) as well as the evolution of emission from supernovae (Chakraborti \& Ray 2011). The dynamical calculations presented here are an obvious essential step in these calculations.

I would like to thank Ramesh Narayan, Lorenzo Sironi and Ralph Wijers for useful discussions.

\section{REFERENCES}

Bianco, C. L. \& Ruffini, R. 2004, ApJ, 605, L1

-. 2005a, ApJ, 620, L23

-. 2005b, ApJ, 633, L13

Blandford, R. D. \& McKee, C. F. 1976, Physics of Fluids, 19, 1130

Chakraborti, S. \& Ray, A. 2011, ApJ, 729, 57

Chevalier, R. A. 1976, ApJ, 207, 872

一. 1982, ApJ, 259, 302

Chiang, J. \& Dermer, C. D. 1999, ApJ, 512, 699

Fender, R. P., Belloni, T. M., \& Gallo, E. 2004, MNRAS, 355, 1105

Fender, R. P., Garrington, S. T., McKay, D. J., Muxlow,

T. W. B., Pooley, G. G., Spencer, R. E., Stirling, A. M., \& Waltman, E. B. 1999, MNRAS, 304, 865

Goodman, J. 1986, ApJ, 308, L47

Granot, J. \& Piran, T. 2012, MNRAS, 421, 570

Granot, J. \& Ramirez-Ruiz, E. 2010, ArXiv e-prints

Hjellming, R. M. \& Rupen, M. P. 1995, Nature, 375, 464

Huang, Y. F., Dai, Z. G., \& Lu, T. 1999, MNRAS, 309, 513

Katz, J. I. \& Piran, T. 1997, ApJ, 490, 772

Lightman, A. P., Press, W. H., Price, R. H., \& Teukolsky, S. A. 1975, Problem book in relativity and gravitation, ed. Lightman, A. P., Press, W. H., Price, R. H., \& Teukolsky, S. A.

Meszaros, P. \& Rees, M. J. 1997, ApJ, 476, 232

Miller-Jones, J. C. A., McCormick, D. G., Fender, R. P., Spencer, R. E., Muxlow, T. W. B., \& Pooley, G. G. 2005, MNRAS, 363, 867
Mirabel, I. F. \& Rodríguez, L. F. 1994, Nature, 371, 46

Narayan, R., Kumar, P., \& Tchekhovskoy, A. 2011, MNRAS, 416, 2193

Narayan, R. \& McClintock, J. E. 2012, MNRAS, 419, L69

Narayan, R., Paczynski, B., \& Piran, T. 1992, ApJ, 395, L83

Paczynski, B. 1986, ApJ, 308, L43

Pe'er, A. \& Wijers, R. A. M. J. 2006, ApJ, 643, 1036

Piran, T. 1999, Phys. Rep., 314, 575

Rees, M. J. \& Meszaros, P. 1992, MNRAS, 258, 41P

Sedov, L. I. 1959, Similarity and Dimensional Methods in Mechanics, ed. Sedov, L. I.

Service, A. T. 1986, ApJ, 307, 60

Shemi, A. \& Piran, T. 1990, ApJ, 365, L55

Shen, R. \& Matzner, C. D. 2012, ApJ, 744, 36

Spitzer, L. 1968, Diffuse matter in space, ed. Spitzer, L.

van Eerten, H. J. \& MacFadyen, A. I. 2012, ApJ, 747, L30

van Paradijs, J., Kouveliotou, C., \& Wijers, R. A. M. J. 2000, ARA\&A, 38, 379

Waxman, E. 1997, ApJ, 491, L19

Xu, M., Nagataki, S., Huang, Y. F., \& Lee, S.-H. 2012, ApJ, 746, 49 